Bull. Austral. Math. Soc.

VOL. 52 (1995) [377-383]

\title{
THE STONE-ČECH COMPACTIFICATION OF Prim $A$
}

\author{
MaY NILSEN
}

For a $C^{*}$-algebra $A$, we give simple proofs of the following: $C_{b}(\operatorname{Prim} A)$ is isomorphic to the centre $Z M(A)$ of the multiplier algebra, $C_{b}(\operatorname{Prim} A)$ is isomorphic to $C(\operatorname{Prim} M(A))$ and Prim $Z M(A)$ is the Stone-Čech compactification of Prim $A$.

\section{INTRODUCTION}

The Dauns-Hofmann Theorem. [2, III Lemma 8.15] Let $A$ be a $C^{*}$-algebra and let Prim $A$ be the primitive ideal space of $A$ with the hull-kernel topology. For each $a \in A$ and $f \in C_{b}(\operatorname{Prim} A)$, there is a unique element $f \cdot a \in A$ such that

$$
f \cdot a-f(P) a \in P \text { for all } P \in \operatorname{Prim} A .
$$

It is easy to see how the Dauns-Hofmann theorem gives an injection from $C_{b}(\operatorname{Prim} A)$ into the centre $Z M(A)$ of the multiplier algebra. It is not evident why this map should be onto. Dixmier [3, Theorem 5(iii)] proved that it was, as did Pedersen [8, Corollary 4.7]. But, we believe that the natural way to view the problem is the reverse of that commonly thought. The easy part is getting the injection from $Z M(A)$ into $C_{b}(\operatorname{Prim} A)$. Then the Dauns-Hofmann theorem is exactly what you need to show that this map is onto (Theorem 1.2). The simplicity of our technique means our proof is much more direct than those given before. An easy corollary of our technique provides an isomorphism from $C_{b}(\operatorname{Prim} A)$ onto $C_{b}(\operatorname{Prim} M(A))$ (Theorem 1.4).

Since Prim $A$ is not necessarily Hausdorff, we carefully define the Stone-Čech compactification and the complete regularisation of such spaces. In Section 2 we deduce from Theorem 1.2 that Prim $Z M(A)$ is the Stone-Čech compactification of Prim $A$, specifying the formula for the canonical mapping (Theorem 2.2). This result is a generalisation of an assertion of Becker [1, p.269]. (We also discuss a problem in Becker's proof.)

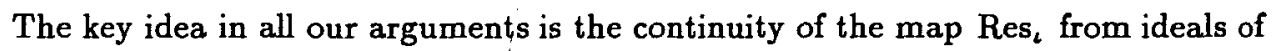
$B$ to ideals of $A$, dual to a homomorphism $\iota: A \rightarrow M(B)$. The map Res ${ }_{\iota}$, and the map

Received 31st January, 1995

This research was supported by the Australian Research Council. I would like to thank my supervisor Iain Raeburn, and his colleague Dana Williams, for their suggestions.

Copyright Clearance Centre, Inc. Serial-fee code: 0004-9729/95 \$A2.00+0.00. 


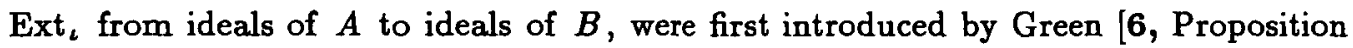
9], and have been predominantly used in the study of crossed products and induced representations [5, Section 2]. However, in this paper we have used these maps in the trivial case; by letting $\iota$ be the identity map, the elementary properties of Res and Ext lead to short, elegant proofs.

In [7], we used Res ${ }_{\iota}$ and Ext $_{\iota}$ to help give a short self-contained proof of the sectional representation theórem [7, Theorem 3.1]. This result says that a $C^{*}$-algebra can be represented as the section algebra of an upper semi-continuous $C^{*}$-bundle over the primitive ideal space of its centre. In [1], Becker asked for a simple necessary and sufficient condition on a $C^{*}$-algebra $A$, which ensures that the $C^{*}$-bundle produced by the sectional representation theorem will be continuous $[1$, p. 268]. This is answered by [7, Theorem 3.1], which says that this happens only when Res ${ }_{j d}$ is open. In Section 3 we show that our condition is equivalent to the condition Becker gave (Proposition 3.1).

\section{DiXMIER'S THEOREM}

Let $A$ be a $C^{*}$-algebra and let $\mathcal{I}(A)$ denote the ideal space of $A$ with the topology which has as subbasic open sets $O_{J}=\{I: I \nsupseteq J\}$, where $J$ is a closed ideal in $A$. The relative topology on $\operatorname{Prim}(A)$ in $\mathcal{I}(A)$ is the usual hull-kernel topology. All representations will be non-degenerate, and the extension of a representation $\rho$ of $A$ to the multiplier algebra $M(A)$ will be denoted by $\bar{\rho}$.

Definitions: Let $A$ and $B$ be $C^{*}$-algebras and $\iota: A \rightarrow M(B)$ a homomorphism. Define $\operatorname{Res}_{\iota}: \mathcal{I}(B) \rightarrow \mathcal{I}(A)$ by $\operatorname{Res}_{\iota}(\operatorname{ker} \sigma)=\operatorname{ker}(\bar{\sigma} \circ \iota)$, where $\sigma$ is a representation of $B$. Define Ext $\iota: \mathcal{I}(A) \rightarrow \mathcal{I}(B)$ by $\operatorname{Ext}_{\iota}(I)=\overline{B \iota(I) B}$ [6, Proposition 9(i)].

In [7, Lemma 1.1] we showed that $\operatorname{Res}_{\iota}(\operatorname{ker} \sigma)=\{a \in A: \iota(a) B \subseteq \operatorname{ker} \sigma\}$. This

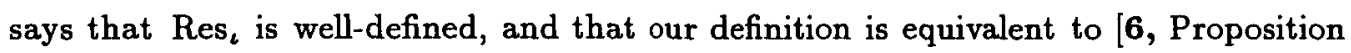
9(i)]. It also implies that Res ${ }_{\iota}$ preserves containment and arbitrary intersections. Using this characterisation, we also showed that $I \subseteq \operatorname{Res}_{\iota}(K) \Leftrightarrow \operatorname{Ext}_{\iota}(I) \subseteq K$. It follows easily from these facts that $\operatorname{Res}_{\iota}$ is continuous [7, Lemma 1.1(iii)], [6, Proposition 9(i)].

Lemma 1.1. Let $A$ be a $C^{*}$-algebra and id: $Z M(A) \rightarrow Z M(A)$ the identity map. Then Resid: Prim $A \rightarrow \operatorname{Prim} Z M(A)$ is a continuous map with dense range, and $\operatorname{Res}_{i d}(\operatorname{ker} \rho)=\operatorname{ker}\left(\left.\bar{\rho}\right|_{Z M(A)}\right)$.

Proof: An irreducible representation $\rho$ of $A$ extends to an irreducible representation $\bar{\rho}$ of $M(A)$. Also $\bar{\rho}(Z M(A))$ is in the centre of $\operatorname{Im} \bar{\rho}$, which is contained in the commutant $\mathbf{C 1}$. Thus $\left.\bar{\rho}\right|_{Z M(A)}$ determines a complex homomorphism, which must be non-zero because $1 \in Z M(A)$. So $\left.\operatorname{ker} \bar{\rho}\right|_{Z M(A)}$ is primitive and Res maps primitive ideals to primitive ideals. The range is dense because $\overline{\operatorname{Res}(\operatorname{Prim} A)}=\{Q: Q \supseteq$ 
$\left.\bigcap_{P} \operatorname{Res}(P)\right\}=\left\{Q: Q \supseteq \operatorname{Res}\left(\bigcap_{P} P\right)\right\}=\operatorname{Prim} Z M(A)$.

Theorem 1.2 DixMIER. Let $A$ be a $C^{*}$-algebra. Then there is an isomorphism $\psi: Z M(A) \rightarrow C_{b}(\operatorname{Prim} A)$ such that $\psi(z)(\operatorname{ker} \rho)=\widehat{z}\left(\operatorname{ker}\left(\left.\bar{\rho}\right|_{Z M(A)}\right)\right)$, where ${ }^{\wedge}$ is the Gelfand transform.

Proof: Define Res id $: C(\operatorname{Prim} Z M(A)) \rightarrow C_{b}(\operatorname{Prim} A)$ by $\operatorname{Res}_{\mathrm{id}}^{*}(g)=g \circ \operatorname{Res}_{\mathrm{id}}$, where $\operatorname{Res}_{i d}$ is as in Lemma 1 . We show that $\operatorname{Res}_{i d}^{*} o^{\wedge}$ is the required isomorphism $\psi$. Since $\operatorname{Res}_{i d}$ has dense range, Res id $^{*}$ is an injection. The Gelfand transform is an isomorphism from $Z M(A)$ onto $C(\operatorname{Prim} Z M(A))$, so it is enough to show that Res id $^{*}$ is onto. Define $L_{f}: A \rightarrow A$ by $L_{f}(a)=f \cdot a$, and $R_{f}: A \rightarrow A$ by $R_{f}(a)=f \cdot a$, where $f \cdot a$ is the unique element given by the Dauns-Hofmann theorem. One can check that $\left(L_{f}, R_{f}\right)$ is a multiplier $z_{f}$ of $A$. Equation (1) implies that $z_{f} a=a z_{f}$ for all $a \in A$, so $z_{f} \in Z M(A)$. Also from (1) we have $\left(z_{f}-f(P)\right) a=f \cdot a-f(P) a \in P$ for all $a \in A$. Since Resid $(P)=\{z \in Z M(A): z A \subset P\}, z_{f}-f(P) 1 \in \operatorname{Res}_{\text {id }}(P)$ for all $P \in \operatorname{Prim} A$. Equivalently, $\widehat{z_{f}}\left(\operatorname{Res}_{\text {id }}(P)\right)=f(P)$, for all $P$. Thus $\operatorname{Res}_{\text {id }}^{*}\left(\widehat{z_{f}}\right)(P)=\widehat{z_{f}}\left(\operatorname{Res}_{\text {id }}(P)\right)=$ $f(P)$, and Res id is an isomorphism.

REMARK. The following lemma is more than we need for Theorem 1.4, but it says that Prim $M(A)$ is a compactification of Prim $A$, in the sense of $[10$, p.1].

Lemma 1.3. Let $A$ be a $C^{*}$-algebra, and $i: M(A) \rightarrow M(A)$ the identity map. Then $\operatorname{Res}_{i}: \operatorname{Prim} A \rightarrow \operatorname{Prim} M(A)$ is a continuous injection with dense range satisfying $\operatorname{Res}_{i}(\operatorname{ker} \rho)=\operatorname{ker} \bar{\rho}$, which is a homeomorphism onto its range.

Proof: If $\rho$ is irreducible the extension $\bar{\rho}$ is also irreducible, so Res $i$ maps between primitive ideal spaces. Suppose $\operatorname{ker} \bar{\rho}=\operatorname{ker} \bar{\sigma}$. Then $\operatorname{ker} \bar{\rho} \circ \iota=\operatorname{ker} \bar{\sigma} \circ \iota$, where $\iota$ is the natural embedding of $A$ into $M(A)$. But $\bar{\rho} \circ \iota=\rho$, and similarly for $\sigma$, so ker $\rho=\operatorname{ker} \sigma$ and $R_{e s} i$ is one-to-one. A calculation similar to that in Lemma 1.1 shows that Res ${ }_{i}$ has dense range. It remains to check that $\operatorname{Res}_{i}$ is open onto its range. In [7, Lemma 1.1(iv)], we showed that Res $i$ is open onto its image if and only if $\left.\operatorname{Ext}_{i}\right|_{\operatorname{Im}(\text { Res })}$ is continuous. So it suffices to show that $\operatorname{Ext}_{i}: \mathcal{I}(M(A)) \rightarrow \mathcal{I}(A)$ is continuous. We have the embedding $\iota$, so define $\operatorname{Res}_{\imath}: \mathcal{I}(M(A)) \rightarrow \mathcal{I}(A)$ as usual. To show $\mathrm{Ext}_{i}$ is continuous we shall show that Ext $_{i}=$ Res $_{\iota}$, that is $\overline{A K A}=\{a: \iota(a) M(A) \subset K\}$. Suppose $\iota(a) m \in K$ for all $m \in M(A)$. Then $\iota(a) \in K$ because $1 \in M(A)$. So $a \in \overline{A K A}$ since $a \sim \mu_{\lambda} \iota(a) \mu_{\lambda}$, where $\mu_{\lambda}$ is an approximate identity in $A$. Conversely, suppose $a, b \in A$ and $k \in K$. Then $\iota(a) k \iota(b) m \in K$ for all $m \in M(A)$. Thus $A K A \subset \operatorname{Res}_{\iota}(K)$, and since $\operatorname{Res}_{\iota}(K)$ is closed, $\overline{A K A}$ is in there too.

ThEOREM 1.4. Let $A$ be a $C^{*}$-algebra. Then there is an isomorphism

$$
\psi: C(\operatorname{Prim} M(A)) \rightarrow C_{b}(\operatorname{Prim} A)
$$


satisfying $\psi(g)(\operatorname{ker} \rho)=g(\operatorname{ker} \bar{\rho})$.

Proof: Lemma 1.3 gives us a continuous map $\operatorname{Res}_{i}: \operatorname{Prim} A \rightarrow \operatorname{Prim} M(A)$, so define $\operatorname{Res}_{i}^{*}: C(\operatorname{Prim} M(A)) \rightarrow C_{b}(\operatorname{Prim} A)$, by $\operatorname{Res}_{i}^{*}(g)=g \circ \operatorname{Res}_{i}$, which is injective because $\operatorname{Res}_{i}$ has dense range. Let $M: Z M(A) \rightarrow Z M(A)$ be the identity map, and define $\operatorname{Res}_{M}: \operatorname{Prim} M(A) \rightarrow \operatorname{Prim} Z M(A)$ as usual. From Lemma 1.1 we have the map $\operatorname{Res}_{\mathrm{id}}: \operatorname{Prim} A \rightarrow \operatorname{Prim} Z M(A)$. From the definitions, $\operatorname{Res}_{\mathrm{id}}=\operatorname{Res}_{M} \circ \operatorname{Res}_{i}$, so that $\operatorname{Res}_{i d}^{*}=\operatorname{Res}_{i}^{*} \circ \operatorname{Res}_{M}^{*}$. By the proof of Theorem 1.2, Res id $^{*}$ is onto, so Res ${ }_{i}^{*}$ must be onto. Choose $\psi=\operatorname{Res}_{i}^{*}$.

\section{StONE-Čech COMPACTIFICATION AND COMPLETE REgUlaRisation}

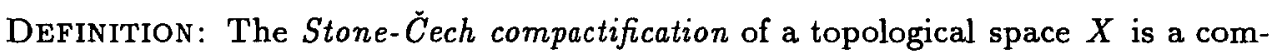
pact Hausdorff space $\beta X$ together with a continuous map $\beta: X \rightarrow \beta X$ such that, for every compact Hausdorff space $Y$ and every continuous map $k: X \rightarrow Y$, there is a unique continuous map $k^{\prime}: \beta X \rightarrow Y$ with $k=k^{\prime} \circ \beta[9,14.1 .1]$.

RemarK. It is well known that $\operatorname{Prim} C_{b}(X)$ is the Stone-Čech compactification of $X$ in the case where $X$ is Hausdorff. The point of the next lemma, is that this is so even when $X$ is not Hausdorff. Although this proof is similar the standard existence proof $[9,14.1 .2]$, this shows how our techniques give a neat exposition.

Lemma 2.1. Let $X$ be a topological space and define $\varepsilon: X \rightarrow \operatorname{Prim} C_{b}(X)$ by $\varepsilon(x)=\operatorname{ker} \varepsilon_{x}$, where $\varepsilon_{x}$ is evaluation at $x$. Then (Prim $\left.C_{b}(X), \varepsilon\right)$ is the Stone-Čech compactification of $X$.

Proof: Let $k: X \rightarrow Y$ be a continuous map, with $Y$ compact Hausdorff. Define $k^{*}: C(Y) \rightarrow C_{b}(X)$ such that $k^{*}(f)=f \circ k$, and define $\operatorname{Res}_{k^{*}}: \mathcal{I}\left(C_{b}(X)\right) \rightarrow \mathcal{I}(C(Y))$ as usual. Lemma 1.1 tells us that it restricts to a map between primitive ideal spaces, so we have $\operatorname{Res}_{k^{*}}: \operatorname{Prim} C_{b}(X) \rightarrow \operatorname{Prim} C(Y)$. Since $Y$ is compact Hausdorff, there exists a homeomorphism $h: \operatorname{Prim} C(Y) \rightarrow Y$. Define $k^{\prime}: \operatorname{Prim} C_{b}(X) \rightarrow Y$ by $k^{\prime}=$ $h \circ \operatorname{Res}_{k^{*}}$. We need to show that $k(x)=k^{\prime}(\varepsilon(x))$ for all $x \in X$. Well, $k^{\prime}(\varepsilon(x))=$ $h \circ \operatorname{Res}_{k^{*}}\left(\operatorname{ker} \varepsilon_{x}\right)=h\left(\operatorname{ker} \varepsilon_{x} \circ k^{*}\right)$, so it suffices to see that $\operatorname{ker} \varepsilon_{k(x)}=\operatorname{ker}\left(\varepsilon_{x} \circ k^{*}\right)$. But $\varepsilon_{x} \circ k^{*}(f)=\varepsilon_{x}(f \circ k)=f \circ k(x)=f(k(x))$, so $f \in \operatorname{ker}\left(\varepsilon_{x} \circ k^{*}\right)$ means $f(k(x))=0$, which is equivalent to $f \in \operatorname{ker}\left(\varepsilon_{k(x)}\right)$. The map $k^{\prime}$ is unique, because the range of $\varepsilon$ is dense in Prim $C_{b}(X)$.

Theorem 2.2. Let $A$ be a $C^{*}$-algebra. Then (Prim $Z M(A), \operatorname{Res}_{i d}$ ) is the Stone-Čech compactification of Prim $A$, where $\operatorname{Res}_{\mathrm{id}}$ : Prim $A \rightarrow \operatorname{Prim} Z M(A)$ satisfies $\operatorname{Res}_{i d}(\operatorname{ker} \rho)=\operatorname{ker}\left(\left.\bar{\rho}\right|_{Z M(A)}\right)$, for all $\operatorname{ker} \rho \in \operatorname{Prim} A$.

Proof: Theorem 1.2 gives an isomorphism $\psi: Z M(A) \rightarrow C_{b}(\operatorname{Prim} A)$, so one can show that $\operatorname{Res}_{\psi}: \operatorname{Prim}\left(C_{b}(\operatorname{Prim} A)\right) \rightarrow \operatorname{Prim} Z M(A)$ is a homeomorphism. Lemma 1.2 
says that for every continuous map $k: \operatorname{Prim} A \rightarrow Y$ ( $Y$ compact Hausdorff), there is a unique continuous map $k^{\prime \prime}: \operatorname{Prim}(C(\operatorname{Prim} A)) \rightarrow Y$ with $k=k^{\prime \prime} \circ \varepsilon$. So define $k^{\prime}: \operatorname{Prim} Z M(A) \rightarrow Y$ by $k^{\prime}=k^{\prime \prime} \circ\left(\operatorname{Res}_{\psi}\right)^{-1}$. It remains to show that $k=k^{\prime} \circ \operatorname{Res}_{\text {id }}$, and for this it is enough to verify $\operatorname{Res}_{\psi} \circ \varepsilon=\operatorname{Res}_{\mathrm{id}}$ :

$$
\begin{aligned}
\operatorname{Res}_{\psi} \circ \varepsilon(\operatorname{ker} \rho) & =\left\{z \in Z M(A): \psi(z) f \in \varepsilon(\operatorname{ker} \rho) \text { for all } f \in C_{b}(\operatorname{Prim} A)\right\} \\
& =\{z: \psi(z) f(\operatorname{ker} \rho)=0 \text { for all } f\} \\
& =\left\{z: \widehat{z}\left(\operatorname{Res}_{\text {id }}(\operatorname{ker} \rho)\right)=0\right\}=\operatorname{Res}_{\text {id }}(\operatorname{ker} \rho) .
\end{aligned}
$$

Thus (Prim $Z M(A), \mathrm{Res}_{\mathrm{id}}$ ) is the Stone-Čech compactification.

Definitions: A topological space $X$ is completely regular if singleton sets are closed and, given a closed set $C$ and a point $x \notin C$, there exists an $f \in C_{b}(X)$ such that $f(x)=0$ and $f(C)=\{1\}[10$, p.4 $]$.

The complete regularisation of a topological space $X$ is a completely regular space $\rho X$ together with a continuous surjection $\rho: X \rightarrow \rho X$ such that, for every completely regular space $Y$ and every continuous surjection $k: X \rightarrow Y$, there is a unique continuous surjection $k^{\prime}: \rho X \rightarrow Y$ with $k=k^{\prime} \circ \rho$.

Lemma 2.3. Let $X$ be a topological space. There is an equivalence relation on $X$ defined by $x_{1} \sim x_{2} \Leftrightarrow f\left(x_{1}\right)=f\left(x_{2}\right)$ for all $f \in C_{b}(X)$, and $(X / \sim, q)$ is the complete regularisation of $X$, where $q: X \rightarrow X / \sim$ is the quotient map.

Proof: Suppose $k: X \rightarrow Y$ is a continuous surjection into a completely regular space. We claim that the map $k^{\prime}: X / \sim \rightarrow Y$ satisfying $k^{\prime}(q(x))=k(x)$ is well defined. Suppose $q\left(x_{1}\right)=q\left(x_{2}\right)$, that is, $f\left(x_{1}\right)=f\left(x_{2}\right)$ for all $f \in C_{b}(X)$. Since $g \circ k \in C_{b}(X)$ for all $g \in C_{b}(Y), g\left(k\left(x_{1}\right)\right)=g\left(k\left(x_{2}\right)\right)$ for all $g$. But $Y$ is completely regular, so $k\left(x_{1}\right)$ must equal $k\left(x_{2}\right)$, and $k^{\prime}$ is well-defined. The map $k^{\prime}$ is continuous by the definition of the quotient topology.

Remarks. 1. When $A$ is unital, Prim $A$ is compact, so the Stone-Cech compactification of Prim $A$ is the complete regularisation by definition. Thus, Theorem 2.2 says that ( $\operatorname{Prim} Z A, \operatorname{Res}_{\mathrm{id}}$ ) is the complete regularisation of $\operatorname{Prim} A$. Since the complete regularisation map is a surjection, when $A$ is unital Resid maps onto Prim $Z A$.

2. Becker proves that Res id is the complete regularisation for unital $A[1, \mathrm{p} .268]$. But he seems to assert that if $P_{1}, P_{2} \in \operatorname{Prim} A$ can be separated by open neighbourhoods in Prim $A$, then there exists $f \in C_{b}(\operatorname{Prim} A)$ such that $f\left(P_{1}\right)=0$ and $f\left(P_{2}\right)=1$. We show that this can fail in general by providing an example of a $C^{*}$-algebra $A$, with $P_{1}$ and $P_{2}$ in Prim $A$ which can be separated, but there exists $P_{3}$ which can't be separated from either $P_{1}$ or $P_{2}$. Thus a function in $C_{b}(\operatorname{Prim} A)$ must agree at $P_{1}$ and $P_{3}$, and at $P_{2}$ and $P_{3}$, and hence agrees at $P_{1}$ and $P_{2}$. This example is due to Dana Williams. 
Let

$$
A=\left\{f \in C\left([0,1], M_{2} \oplus M_{2}\right): f(1)=\left(\begin{array}{ll}
\alpha & 0 \\
0 & \beta
\end{array}\right) \oplus\left(\begin{array}{ll}
\beta & 0 \\
0 & \gamma
\end{array}\right), \text { for some } \alpha, \beta, \gamma \in \mathbf{C}\right\}
$$

Let $p_{1}$ and $p_{2}$ be the projections id $\oplus 0$ and $0 \oplus$ id respectively. Then for each $t \in[0,1)$, let $\pi_{i}^{t}(f)=p_{i} f(t)$. We also have representations defined by $\pi_{x}(f)=\alpha, \pi_{y}(f)=\beta$ and $\pi_{z}(f)=\gamma$, where $f(1)=\left(\begin{array}{ll}\alpha & 0 \\ 0 & \beta\end{array}\right) \oplus\left(\begin{array}{ll}\beta & 0 \\ 0 & \gamma\end{array}\right)$. These are all the irreducible representations. Note that as $t \rightarrow 1, \pi_{1}^{t}$ converges to both $\pi_{x}$ and $\pi_{y}$, while $\pi_{2}^{t}$ converges to both $\pi_{y}$ and $\pi_{z}$. It follows that neither $\pi_{x}$ and $\pi_{y}$, nor $\pi_{y}$ and $\pi_{z}$, can be separated by disjoint open sets. Since $A$ is a Type I $C^{*}$-algebra, neither $\operatorname{ker} \pi_{x}$ and $\operatorname{ker} \pi_{y}$, nor $\operatorname{ker} \pi_{y}$ and $\operatorname{ker} \pi_{z}$, can be separated by disjoint open sets. Now let $I_{1}=\left\{f \in A: p_{2} f(t)=0\right.$ for all $\left.t \in[0,1]\right\}$, and similarly for $I_{2}$. Then $O_{I_{i}}=$ $\left\{Q \in \operatorname{Prim} A: Q \not \supset I_{i}\right\}$, for $i=1,2$, are disjoint open sets with $\operatorname{ker} \pi_{x} \in O_{I_{1}}$ and $\operatorname{ker} \pi_{z} \in O_{I_{2}}$. Thus $\operatorname{ker} \pi_{x}$ and $\operatorname{ker} \pi_{z}$ can be separated.

\section{BECKER ON SECTIONAL REPRESENTATION}

Becker asks for a simple condition on $A$ which ensures that the the $C^{*}$-bundle produced by the sectional representation theorem is continuous. From [7, Theorem 3.1] we know that this happens only when $\operatorname{Res}_{\text {id }}: \operatorname{Prim} A \rightarrow \operatorname{Prim} Z M(A)$ is open. We shall now show that our condition is equivalent to the condition Becker gives $[1, p .268]$.

Proposition 3.1. Let $A$ be a $C^{*}$-algebra and id: $Z M(A) \rightarrow Z M(A)$ be the identity map. The map $\operatorname{Res}_{\mathrm{id}}: \operatorname{Prim} A \rightarrow \operatorname{Prim} Z M(A)$ is open, if and only if

$$
I \supseteq \bigcap\left\{J \in \operatorname{Prim} Z M(A): a \in \operatorname{Ext}_{\mathrm{id}}(J)\right\} \Rightarrow a \in \operatorname{Ext}_{\mathrm{id}}(I),
$$

where $a \in A$ and $I \in \operatorname{Prim} Z M(A)$.

Proof: Suppose Resid is open and $I \supseteq \bigcap\{J: a \in \operatorname{Ext}(J)\}$. Dixmier says $\{P \in \operatorname{Prim} A: a \notin P\}$ is open in Prim $A[4,3.1 .2]$. Since Res is open, $\{\operatorname{Res}(P): a \notin P\}$ is open in $\operatorname{Prim} Z M(A)$. We claim that this open set is equal to $\{J: a \notin \operatorname{Ext}(J)\}$. Suppose $J$ is in the first set. That is, there exists a $P$ such that $J=\operatorname{Res}(P)$ and $a \notin P$. This is the same as $P \supseteq \operatorname{Ext}(J)$ and $a \notin P$, and this implies $a \notin \operatorname{Ext}(J)$. Conversely, suppose $J$ is in the second set. That means $a \notin \operatorname{Ext}(J)$. So, there must exist at least one primitive ideal $P \supseteq \operatorname{Ext}(J)$ such that $a \notin P$, because every ideal is the intersection of all primitive ideals containing it. But $P \supseteq \operatorname{Ext}(J)$ is equivalent to $\operatorname{Res}(P)=J$ (since $J$ is maximal), so $J$ is in the first set. Thus we have that $\{J: a \in \operatorname{Ext}(J)\}$ is closed. Since $I \supseteq \bigcap\left\{J: a \in \operatorname{Ext}_{\text {id }}(J)\right\}, I$ is in the closure of the set, so $I$ is actually an element of it, which means that $a \in \operatorname{Ext}(I)$. 
Conversely, suppose $I \supseteq \bigcap\left\{J \in \operatorname{Prim} Z M(A): a \in \operatorname{Ext}_{\text {id }}(J)\right\} \Rightarrow a \in \operatorname{Ext}_{\text {id }}(I)$. We begin by proving $I_{\lambda} \rightarrow I$ in $\operatorname{Im}\left(\operatorname{Res}_{\mathrm{id}}\right)$ implies $\operatorname{Ext}(I) \supseteq \bigcap_{\lambda} \operatorname{Ext}\left(I_{\lambda}\right)$. Suppose $I_{\lambda} \rightarrow I$ and $a \in \operatorname{Ext}\left(I_{\lambda}\right)$ for all $\lambda$. Then $I_{\lambda} \in\{J: a \in \operatorname{Ext}(J)\}$ for all $\lambda$, so $I \supseteq \bigcap_{\lambda} I_{\lambda} \supseteq \bigcap\{J: a \in \operatorname{Ext}(J)\}$. By the assumption then, $a \in \operatorname{Ext}(I)$ and $\operatorname{Ext}(I) \supseteq$ $\bigcap_{\lambda} \operatorname{Ext}\left(I_{\lambda}\right)$.

To show Res is open, its enough to show that $\operatorname{Comp} \operatorname{Res}\left(O_{K}\right)$ is closed. Suppose $I_{\lambda} \rightarrow I$ in $\operatorname{Im}(\operatorname{Res})$ and $I_{\lambda} \in \operatorname{Comp} \operatorname{Res}\left(O_{K}\right)$ for all $\lambda$. That means $\operatorname{Ext}\left(I_{\lambda}\right) \in$ $\operatorname{Comp}\left(O_{K}\right)$ for all $\lambda$ (since $J \in \operatorname{Res}\left(O_{K}\right) \Leftrightarrow \operatorname{Ext}(J) \in O_{K}$ [7, Equation 2]). That is, $\operatorname{Ext}\left(I_{\lambda}\right) \supseteq K$ for all $\lambda$, so $\bigcap_{\lambda} \operatorname{Ext}\left(I_{\lambda}\right) \supset K$, and then we have $\operatorname{Ext}(I) \supseteq K$. Hence $I \in \operatorname{Comp} \operatorname{Res}\left(O_{K}\right)$ and $\operatorname{Comp} \operatorname{Res}\left(O_{K}\right)$ is closed.

\section{REFERENCES}

[1] T.Becker, 'A few remarks on the Dauns-Hofmann theorems for $C^{*}$-algebras', Arch. Math. 43 (1984), 265-269.

[2] J. Dauns and K.H. Hofmann, 'Representations of rings by sections', Mem. Amer. Math. Soc. 83 (1968).

[3] J. Dixmier, 'Ideal center of a $C^{*}$-algebra', Duke Math. J. 35 (1968), 375-382.

[4] J. Dixmier, $C^{*}$-algebras (North-Holland, New York, 1977).

[5] E.C. Gootman and A.J. Lazar, 'Crossed products of type I AF algebras by abelian groups', Israel J. Math. 56 (1986), 267-279.

[6] P. Green, 'The local structure of twisted covariance algebras', Acta Math. 140 (1978), 191-250.

[7] M. Nilsen, ' $C$ *-bundles', (preprint, 1995).

[8] G. Pedersen, 'Applications of weak* semicontinuity in $C^{*}$-algebra theory', Duke Math. J. 39 (1972), 431-450.

[9] Z. Semadeni, Banach spaces of continuous functions, Volume 1 (PWN, Polish Scientific, Warsaw, 1971).

[10] R.C. Walker, The Stone-Cech compactification (Springer Verlag, Berlin, Heidelberg, New York, 1974).

Department of Mathematics

The University of Newcastle

Callaghan NSW 2308

Australia

e-mail: may@frey.newcastle.edu.au 